\title{
Time machines: the Principle of Self-Consistency as a consequence of the Principle of Minimal Action
}

\author{
A. Carlini \\ NORDITA, Blegdamsvej 17, DK-2100 Copenhagen Ø, Denmark \\ V. P. Frolov \\ Theoretical Physics Institute, University of Alberta, Edmonton, Canada T6G 2J1 \\ P.N. Lebedev Physical Institute, Leninsky Prospect 53, Moscow, Russia \\ M. B. Mensky \\ P.N. Lebedev Physical Institute, 117924, Moscow, Russia \\ TAC, Blegdamsvej 17, DK-2100 Copenhagen Ø, Denmark \\ I. D. Novikov \\ The Copenhagen University Observatory, Østervoldgade 3, DK-1350 Copenhagen K, \\ Denmark \\ NORDITA, Blegdamsvej 17, DK-2100 Copenhagen Ø, Denmark \\ TAC, Blegdamsvej 17, DK-2100 Copenhagen Ø, Denmark \\ Astro Space Center of the P.N. Lebedev Physical Institute, Profsoyuznaja 84/32, Moscow, \\ 117810, Russia \\ H. H. Soleng \\ Theory Division, CERN, CH-1211 Geneva 23, Switzerland
}




\begin{abstract}
We consider the action principle to derive the classical, non-relativistic motion of a self-interacting particle in a 4-D Lorentzian spacetime containing a wormhole and which allows the existence of closed time-like curves. For the case of a 'hardsphere' self-interaction potential we show that the only possible trajectories (for a particle with fixed initial and final positions and which traverses the wormhole once) minimizing the classical action are those which are globally self-consistent, and that the 'Principle of self-consistency' (originally introduced by Novikov) is thus a natural consequence of the 'Principle of minimal action.'
\end{abstract}




\section{Introduction}

The possibility that the laws of physics might allow for the existence of closed timelike curves (CTCs) inside our universe has been a long time conjecture [1], which has been more recently revived by a series of papers [2-15]. Macroscopic CTCs might be easily realized as a semiclassical consequence of the 'quantum foam' structure of spacetime at Planck scales (see, e.g., Ref. [16]). The idea that 4-d geometry itself might be no longer a fundamental concept, and that close to the Planck scale one should instead allow for a 'quantum fuzz' in which spacetime continuosly undergoes non-trivial topological fluctuations, was first introduced by Wheeler [17].

One peculiar kind of these topological fluctuations is the so-called wormhole, intuitively speaking a 4-d 'handle-like' geometry, whose two 'mouths' join distant regions of spacetime. Provided the matter density in some regions of our universe satisfies certain properties (i.e. it violates the so-called 'averaged weak energy conditions,' see, e.g., Refs. [3-6]), these 4-d Lorentzian wormhole geometries could, in principle, exist (at least they can be exact solutions of the Einstein equations).

It has then been shown $[4,7-8]$ that generic relative motions of the two wormhole's mouths, or equivalently generic gravitational redshifts at the mouths due to external gravitational fields, can indeed in principle produce CTCs: if the wormhole is traversed from mouth to mouth, it acts as a 'time machine' allowing one to travel into the past or into the future.

For spacetimes with CTCs, past and future are no longer 'globally' distinct, and the (Cauchy) problem of evolving the equations of motion of a particle (or field) from a set of initial conditions into the 'future' is in general more involved than for spacetimes without CTCs $[9,11,12]$. In particular, as originally pointed out in Ref. [13], events on CTCs should causally influence each other along the 'loops in time' in a self-adjusted, consistent way. This requirement has been explicitly formulated as the 'Principle of self-consistency,' according to which the only solutions to the laws of physics that can occur locally in the real universe are those which are globally self-consistent [7, 9, 13-15].

The question arises whether the 'Principle of self-consistency' really is an independent assumption which is necessary in order to make sense of the spacetimes with CTCs without resorting to 'new physics,' or whether it actually can be incorporated into some other, more fundamental, physical principle.

In this paper we consider, in the framework of the action principle, the problem of the classical, non-relativistic motion of a self-interacting point particle, passing once trough a wormhole 'time machine.' For this idealized model we are able to show that the 'Principle of self-consistency' is in fact a direct consequence of the 'Principle of minimal action.'

In particular, in Section 2 we introduce the main formulas for the kinematics, the dynamical equations and conservation laws for the case of a general, central self-interaction potential, and state the main lines for the analysis of the stationary 
points of the action describing the classical motion of the particle in three spatial dimensions. In Section 3 we turn to the more specific case of a 'hard-sphere' selfinteraction potential (effectively treating the particle as a 'billiard ball'), and we separately analyze the classical motions for the cases without and with collisions. We show (explicit and detailed formulas for the case of a coplanar motion of the particle with respect to the wormhole's mouths are presented in the appendix A.2) that the action is minimized along all these trajectories, and therefore conclude that the globally self-consistent solutions for our model are a direct consequence of the principle of minimal action. We conclude in Section th with some remarks on the reformulation of the model in terms of a Cauchy initial problem and compare with results presented in previous literature.

\section{The model}

We consider the motion of a self-interacting particle of mass $m$ in the background with a wormhole 'time machine'. The mouths of the wormhole are assumed to have a size which is much smaller than any other scale present in the model, so that they can be treated as pointlike, and to be infinitely heavy, so that we can neglect the recoil effect on the geometry when the particle traverses the wormhole. In particular, we suppose that the mouths are at rest in some reference frame, and consider the problem using this frame. Moreover, spacetime outside the 'time machine' is approximated to be Minkowskian, and the motion of the particle to be non-relativistic. We further restrict our analysis to motions in which the particle traverses the wormhole only once. Our discussion will be essentially independent of other features defining the internal structure of the wormhole (although it is consistent, e.g., with the choice of 'traversal rules' suggested in Ref. [11]).

The motion can be schematically described in the following way. The particle is assumed to start at time $t_{i}$ in the position $\vec{r}_{i}$, to enter the first mouth (B) of the wormhole at time $\bar{t}+\tau$ (position $\vec{r}_{B}$ ), to exit from the other mouth (A) at the earlier time $\bar{t}$ (position $\vec{r}_{A}$ ) and to finally end its trajectory at time $t_{f}$ in the position $\vec{r}_{f}$. For the particle itself (in its proper time), the motion through the wormhole happens almost istantaneously, as the path length of the wormhole handle is assumed to be infinitely short. According to an external observer, instead, the particle traversing the time machine travels back in time by the amount $\Delta t=-\tau$, where by definition $\tau>0$.

We consider the case ${ }^{\natural}$

$$
\begin{aligned}
& \bar{t}>t_{i} \\
& t_{f}>\bar{t}+\tau .
\end{aligned}
$$

We first analyze the generic motion of the particle in three spatial dimensions, and

${ }^{a}$ It is also possible to consider other relations among the times $t_{i}, t_{f}, \bar{t}$, and $\tau$, but for simplicity we shall not do this here. 
then discuss the detailed trajectories for the case of coplanar motion with respect to the wormhole's mouths.

Between times $\bar{t}$ and $\bar{t}+\tau$ there are two copies of the same particle, which are treated as independent objects subject to an interaction potential $V$ of central type. The motion can be then divided into three main regions:

I) $t_{i}<t<\bar{t}$ : only the first copy of the particle with position $\vec{r}_{1}(t)$ is present;

II) $\bar{t}<t<\bar{t}+\tau$ : both copies of the particle with positions $\vec{r}_{1}(t)$ and $\vec{r}_{2}(t)$ are present;

III) $\bar{t}+\tau<t<t_{f}$ : only the second copy of the particle with position $\vec{r}_{2}(t)$ is present.

We assume the initial and final position of the particle

$$
\begin{aligned}
& \vec{r}_{1}\left(t_{i}\right) \equiv \vec{r}_{i} \\
& \vec{r}_{2}\left(t_{f}\right) \equiv \vec{r}_{f}
\end{aligned}
$$

to be fixed.

The time delay $\tau$ in the wormhole, as well as the positions of the wormhole's mouths are also assumed to be known, and we neglect the interaction between the particle and the mouths. The entrance and exit conditions on the position of the particle are formally summarized as the constraints

$$
\begin{aligned}
& \vec{r}_{1}(\bar{t}+\tau)=\vec{r}_{B} \\
& \vec{r}_{2}(\bar{t})=\vec{r}_{A} .
\end{aligned}
$$

The total action describing such a motion is the sum of the actions of the single paths in each separate region (subject to obvious continuity conditions for the position of the copies of the particle at times $\bar{t}$ and $\bar{t}+\tau$ ), i.e.,

$$
\begin{aligned}
S= & \frac{m}{2} \int_{t_{i}}^{\bar{t}} d t{\dot{\overrightarrow{r_{1}}}}^{2}(t)+\frac{m}{2} \int_{\bar{t}+\tau}^{t_{f}} d t{\dot{\overrightarrow{r_{2}}}}^{2}(t)+\int_{\bar{t}}^{\bar{t}+\tau} d t\left\{\frac{m}{2} \dot{\vec{r}}_{1}^{2}(t)+\frac{m}{2} \dot{\vec{r}}_{2}^{2}(t)\right. \\
& \left.-V\left(\left|\vec{r}_{1}(t)-\vec{r}_{2}(t)\right|\right)\right\} \equiv S_{1}\left(t_{i}, \bar{t}\right)+S_{2}\left(\bar{t}+\tau, t_{f}\right)+S_{12}(\bar{t}, \bar{t}+\tau) .
\end{aligned}
$$

The general procedure consists in imposing the principle of stationarity of the action, deriving the classical equations of motion in each of the three Regions I, II, and III, and solving them separately. In the variational principle we only consider continuous paths for which the initial and final positions are held fixed and subject to conditions (3).

Regions I and III. By the variation of the action $S_{1}$ of Eq. (蛋) with respect to $\vec{r}_{1}$ in the first region and $S_{2}$ with respect to $\vec{r}_{2}$ in the third region, we find

$$
\begin{array}{lll}
\frac{\delta S_{1}}{\delta \vec{r}_{1}}=0 \quad & \Rightarrow \quad m \ddot{\overrightarrow{r_{1}}}=0, \\
\frac{\delta S_{2}}{\delta \overrightarrow{r_{2}}}=0 \quad \Rightarrow \quad m \ddot{\overrightarrow{r_{2}}}=0 .
\end{array}
$$

Equations (5) clearly represent linear motion. 
Region II. In this region the motion is more involved, as the two copies of the particle interact via the potential $V$.

Let us consider the case of a general potential first. By varying the action $S_{12}$ with respect to $\vec{r}_{1}$ and $\vec{r}_{2}$ we have the following equations of motion

$$
\begin{array}{lll}
\frac{\delta S_{12}}{\delta \vec{r}_{1}}=0 & \Rightarrow \quad m \ddot{\vec{r}_{1}}=V^{\prime}\left(\left|\vec{r}_{2}-\vec{r}_{1}\right|\right) \frac{\left(\vec{r}_{2}-\vec{r}_{1}\right)}{\left|\vec{r}_{2}-\vec{r}_{1}\right|}, \\
\frac{\delta S_{12}}{\delta \vec{r}_{2}}=0 & \Rightarrow \quad m \ddot{\vec{r}_{2}}=-V^{\prime}\left(\left|\vec{r}_{2}-\vec{r}_{1}\right|\right) \frac{\left(\vec{r}_{2}-\vec{r}_{1}\right)}{\left|\vec{r}_{2}-\vec{r}_{1}\right|} .
\end{array}
$$

For a general, position-dependent potential $V$, it is obvious from Eqs. (6) that the motion will no longer be linear.

We can simplify the analysis by introducing the two variables

$$
\begin{aligned}
& \vec{r}(t) \equiv \vec{r}_{2}(t)-\vec{r}_{1}(t) \\
& \vec{R}(t) \equiv \frac{1}{2}\left[\vec{r}_{1}(t)+\vec{r}_{2}(t)\right]
\end{aligned}
$$

Summing and subtracting Eqs. (6) we therefore have

$$
\begin{aligned}
& m \ddot{\vec{R}}(t)=0, \\
& m \ddot{\vec{r}}(t)=-2 V^{\prime}[r(t)] \frac{\vec{r}}{r},
\end{aligned}
$$

where we have defined $r \equiv|\vec{r}|$. The new equations (8) admit the integrals

$$
\begin{aligned}
& \vec{U}=\dot{\vec{R}} \\
& \mathcal{E}=\frac{1}{2} m \dot{\vec{r}}^{2}(t)+2 V[r(t)]
\end{aligned}
$$

where $\vec{U}=$ const and $\mathcal{E}=$ const. Equations (9) are nothing but the laws of energy and momentum conservation for the motion of the two copies of the particle in Region II. We incidentally note that the same integrals could have been derived in a standard way via the variational principle, by simply noting that the action (何) is invariant with respect to translations in both time and space directions.

For a generic, long-range potential $V$, the trajectories will therefore depend on the parameters

$$
\bar{t}, \quad \vec{r}_{1}(\bar{t}) \equiv \vec{r}_{10}, \quad \vec{r}_{2}(\bar{t}+\tau) \equiv \vec{r}_{20} .
$$

The general method is then to verify whether there is a value (at least one) of such parameters for which the total action (4) evaluated along such trajectories has a minimum (at least local). This would imply that the only possible classical trajectories for the particle for which the action is stationary and minimized are those which are globally self-consistent. It is in this sense that the 'Principle of self-consistency' [7, 9, 13-15], can be looked at as a direct consequence of the 'Principle of minimal action.'

\footnotetext{
${ }^{b}$ For a generic motion in three spatial dimensions, the number of parameters is seven, i.e. $\bar{t}$ plus the three components of $\vec{r}_{10}$ and $\vec{r}_{20}$.
} 
In general, the problem of solving the equations of motion and minimizing the classical action for a generic potential $V$ is not straightforward. In the case, e.g., of a Coulomb-like repulsive interaction between the two copies of the particle, the problem can be shown to finally reduce to that of looking for the stationary points of the classical action with respect to the parameters (eccentricity, semimajor axis etc.) of the hyperbolic orbit for $\vec{r}$. The system of equations involved in the procedure, however, cannot be solved exactly in an analytic form, but only by making some specific ansatz and using, e.g., some perturbative expansion. We hope to turn back to this case in a future work.

\section{The case of a 'hard-sphere' potential}

The nature of the trajectories can be greatly simplified, instead, by working with a 'short-range' potential. We can assume, for instance, that the two copies of the particle are like two (small) 'billiard balls,' interacting via a hard-sphere potential of the kind

$$
V(r)=V_{1} \theta\left(r_{s}-r\right) ; \quad V_{1} \rightarrow \infty, \quad r_{s} \rightarrow 0 .
$$

In this approximation, we essentially neglect the interaction of the two copies of the particle along most of their motion in Region II, except at the point of the (eventual) collision, which we assume to be essentially elastic (we also neglect the deformation of the 'balls'). The effect of the potential is limited to an infinitesimally small period of time around $t_{0}$, the time of the (eventual) collision.

For such a potential, it is clear that the second of Eqs. (8) and (9) are not even well defined at the point $r=r_{s}$. However, also in this case it is still possible to

show (see Appendix A.1) that the kinetic energy $\dot{\vec{r}}^{2}$ is conserved before and after the collision.

The total momentum is also conserved during the whole motion in Region II (and also before and after the eventual collision point), as clearly the first of Eqs. (9) shows.

Finally, taking the variation of the action (四) with respect to $\bar{t}$, and excluding the possibility of collisions on the verge of the wormhole's mouths (in other words, if we assume that, for the short-range potential (11), $V[r(\bar{t})]=V[r(\bar{t}+t)]=0$ ), we get the condition

$$
\left[\dot{\vec{r}}_{1}(\bar{t}+\tau)\right]^{2}=\left[\dot{\vec{r}}_{2}(\bar{t})\right]^{2}
$$

stating that the energy of the particle at its entrance and exit from the wormhole's mouths must be conserved. The condition (12) had also been identified by the authors of Ref. [11] as one of the wormhole 'traversal rules'.

Moreover, for $r>r_{s} \sim 0$ we have $V(r)=0$, and Eqs. (6) are now perfectly well defined: they state that, everywhere in Region II except at the point of eventual collision, the motion of the two copies of the particle is also linear.

In conclusion, for fixed initial and final positions of a particle constrained to traverse the wormhole once, we have to distinguish between the two cases: 
i) trajectories without self-collision. In this case, the first copy of the particle moves linearly from the initial position $\vec{r}_{i}$ at time $t_{i}$ until it enters the wormhole mouth B at time $t=\bar{t}+\tau$. Similarly, the second copy of the particle moves linearly from the mouth A at time $\bar{t}$ up to the final position $\vec{r}_{f}$ at time $t_{f}$.

ii) trajectories with self-collision. In this case, instead, the motion for the first (second) copy of the particle is linear from the initial position $\vec{r}_{i}$ at time $t_{i}$ (from the wormhole mouth $\mathrm{A}$ at time $\bar{t}$ ) up to the collision event, with coordinates

$$
\vec{r}_{1}\left(t_{0}\right)=\vec{r}_{2}\left(t_{0}\right) \equiv \vec{r}_{0} .
$$

After the collision, the motion of the first (second) copy of the particle is linear again up to the wormhole mouth B at time $\bar{t}+\tau$ (up to the final position $\vec{r}_{f}$ at time $t_{f}$ ). Of course, the directions of the motions for the first (second) copy of the particle will be, in general, different before and after the collision (see Section 3.2).

In the case of a short-range potential, therefore, the stationarity problem is easier than for the general potential case. The trajectories will depend, in fact, only on the parameters $\mathrm{H}$

$$
\bar{t}, t_{0}, \quad \vec{r}_{0} .
$$

The problem is now to look for the stationary points (if any) of the action (4), evaluated along the classical trajectories (5), (6), and (8), with respect to the parameters (14), and to see if they are minima. We first consider in details the variational problem for the case of no collision (i.e., when the separation $r$ between the two copies of the particle is always greater than $r_{s}$ ), so as to show how the main lines of the analysis proceed.

\subsection{Trajectories without self-collision}

In the no-collision case, the solutions of the equations of motion (5)-(6), subject to the boundary conditions (2)-(3), are given by the linear trajectories

$$
\begin{aligned}
& \vec{r}_{1}(t ; \bar{t})=\frac{\left[\vec{r}_{B}\left(t-t_{i}\right)+\vec{r}_{i}(\bar{t}+\tau-t)\right]}{\left(t+\tau-t_{i}\right)}, \\
& \vec{r}_{2}(t ; \bar{t})=\frac{\left[\vec{r}_{A}\left(t_{f}-t\right)+\vec{r}_{f}(t-\bar{t})\right]}{\left(t_{f}-\bar{t}\right)} .
\end{aligned}
$$

The variational problem is now extremely simple, as the action (4i) evaluated along the classical trajectories (15) is a function only of the parameter $\bar{t}$. Noting that for these classical trajectories, the contribution of the potential term to the action (困) is identically zero (see Appendix A.1), and using Eqs. (15) for the kinetic terms, the classical action for the no-collision case $\left(S_{c l, n-c}\right)$ becomes

$$
S_{c l, n-c}(\bar{t})=\frac{m}{2}\left[\frac{\left(\vec{r}_{B}-\vec{r}_{i}\right)^{2}}{\left(\bar{t}+\tau-t_{i}\right)}+\frac{\left(\vec{r}_{f}-\vec{r}_{A}\right)^{2}}{\left(t_{f}-\bar{t}\right)}\right] .
$$

${ }^{c}$ In three spatial dimensions, these are five 'degrees of freedom' instead of the seven of the general case. 
It is now easy to see that variation of Eq. (16) with respect to $\bar{t}$ gives again condition (12) for the case of the trajectories (15). Solving Eq. (12) for $\bar{t}$ we get

$$
\bar{t}=\frac{\left[t_{f} \pm\left(t_{i}-\tau\right) W\right]}{(1 \pm W)}
$$

where we have denoted $W \equiv\left|\vec{r}_{f}-\vec{r}_{A}\right| /\left|\vec{r}_{B}-\vec{r}_{i}\right|$.

We have to check that in finding the two stationary solutions (17) for $\bar{t}$ we have not inadvertently violated the conditions (1), i.e. that

$$
t_{i}<\bar{t}<t_{f}-\tau
$$

It is easy to see that the only solution satisfying the condition (18) is given by Eq. (17) with the plus sign, subject to the constraint that

$$
t_{f}-t_{i}>\max \left\{W \tau ; \quad W^{-1} \tau\right\} .
$$

Substituting Eq. (17) back into Eqs. (15) finally gives the explicit trajectories

$$
\begin{aligned}
& \vec{r}_{1}(t)=\vec{r}_{i}+(1+W)\left(\vec{r}_{B}-\vec{r}_{i}\right) \frac{\left(t-t_{i}\right)}{\left(t_{f}-t_{i}+\tau\right)}, \\
& \vec{r}_{2}(t)=\vec{r}_{f}+\left(1+W^{-1}\right)\left(\vec{r}_{f}-\vec{r}_{A}\right) \frac{\left(t-t_{f}\right)}{\left(t_{f}-t_{i}+\tau\right)} .
\end{aligned}
$$

The stationary point (17) is also clearly a minimum of the action $S_{c l, n-c}$, since

$$
\left.\frac{d^{2} S_{c l, n-c}}{d \bar{t}^{2}}\right|_{s t a t}=m\left[\frac{\left(\vec{r}_{B}-\vec{r}_{i}\right)^{2}}{\left(\bar{t}+\tau-t_{i}\right)^{3}}+\frac{\left(\vec{r}_{f}-\vec{r}_{A}\right)^{2}}{\left(t_{f}-\bar{t}\right)^{3}}\right]>0 .
$$

We conclude that the problem of a 'billiard ball'-like particle moving between specified initial and final positions, subject to the conditions of traversing the wormhole once and having no self-collisions, has a unique globally self-consistent solution which can be derived by simply imposing the principle of minimal action.

\subsection{Trajectories with self-collision}

In the case of self-collision under the action of the 'hard-sphere' potential (11), as we already remarked at the beginning of Section 2, the motion is also linear everywhere except at the event of the impact. To clearly identify the trajectories before and after the collision, it is convenient to slightly modify the notation defining

$$
\begin{array}{ll}
\vec{r}_{1}(t), & t_{i}<t<t_{0}, \\
\vec{r}_{1}^{\prime}(t), & t_{0}<t<\bar{t}+\tau,
\end{array}
$$

for the first copy of the particle, and

$$
\begin{array}{ll}
\vec{r}_{2}(t), & \bar{t}<t<t_{0}, \\
\vec{r}_{2}^{\prime}(t), & t_{0}<t<t_{f},
\end{array}
$$


for the second copy of the particle.

It is then easy to show that the solutions of the classical equations of motion (8) -(6), subject to the boundary conditions (22)-(3), and (13), are given by

$$
\begin{aligned}
& \vec{r}_{1}\left(t ; \bar{t}, t_{0}, \vec{r}_{0}\right)=\frac{\left[\vec{r}_{0}\left(t-t_{i}\right)+\vec{r}_{i}\left(t_{0}-t\right)\right]}{\left(t_{0}-t_{i}\right)}, \\
& \vec{r}_{2}\left(t ; \bar{t}, t_{0}, \vec{r}_{0}\right)=\frac{\left[\vec{r}_{0}(t-\bar{t})+\vec{r}_{A}\left(t_{0}-t\right)\right]}{\left(t_{0}-\bar{t}\right)}, \\
& \vec{r}_{1}^{\prime}\left(t ; \bar{t}, t_{0}, \vec{r}_{0}\right)=\frac{\left[\vec{r}_{B}\left(t-t_{0}\right)+\vec{r}_{0}(\bar{t}+\tau-t)\right]}{\left(\bar{t}+\tau-t_{0}\right)} \\
& \vec{r}_{2}^{\prime}\left(t ; \bar{t}, t_{0}, \vec{r}_{0}\right)=\frac{\left[\vec{r}_{f}\left(t-t_{0}\right)+\vec{r}_{0}\left(t_{f}-t\right)\right]}{\left(t_{f}-t_{0}\right)}
\end{aligned}
$$

With the new notation (22)-(23), the action (14) more simply reads

$$
\begin{aligned}
\hat{S}= & \frac{m}{2}\left[\int_{t_{i}}^{t_{0}} d t\left[\dot{\overrightarrow{r_{1}}}(t)\right]^{2}+\int_{t_{0}}^{\bar{t}+\tau} d t\left[\dot{\vec{r}}_{1}^{\prime}(t)\right]^{2}+\int_{\bar{t}}^{t_{0}} d t\left[\dot{\overrightarrow{r_{2}}}(t)\right]^{2}\right. \\
& \left.+\int_{t_{0}}^{t_{f}} d t\left[\dot{\vec{r}}_{2}^{\prime}(t)\right]^{2}\right]-\int_{\bar{t}}^{\bar{t}+\tau} d t V\left(\left|\vec{r}_{1}(t)-\vec{r}_{2}(t)\right|\right)
\end{aligned}
$$

In order to evaluate the total action $\hat{S}_{c l, c}$ for the collision case along the classical trajectories described by Eqs. (24), we can use arguments similar to those in Section 3.1 (see Appendix A.1) to show that the contribution of the potential term is again zero.

Therefore, using the classical trajectories (24) for the kinetic terms in Eq. (25), we obtain

$$
\hat{S}_{c l, c}\left(\bar{t}, t_{0}, \vec{r}_{0}\right)=\frac{m}{2}\left[\frac{\left(\vec{r}_{0}-\vec{r}_{i}\right)^{2}}{\left(t_{0}-t_{i}\right)}+\frac{\left(\vec{r}_{0}-\vec{r}_{B}\right)^{2}}{\left(\bar{t}+\tau-t_{0}\right)}+\frac{\left(\vec{r}_{0}-\vec{r}_{A}\right)^{2}}{\left(t_{0}-\bar{t}\right)}+\frac{\left(\vec{r}_{0}-\vec{r}_{f}\right)^{2}}{\left(t_{f}-t_{0}\right)}\right] .
$$

The variational problem for the collision case consists in looking for the stationary points of the action (26) with respect to the parameters $\vec{r}_{0}, t_{0}$, and $\bar{t}$. Taking derivatives with respect to $\overrightarrow{r_{0}}, t_{0}$, and $\bar{t}$, and defining the velocities $\vec{v}_{1} \equiv \dot{\vec{r}}_{1}, \vec{v}_{2} \equiv \dot{\vec{r}}_{2}, \vec{v}_{1}^{\prime} \equiv \dot{\vec{r}}_{1}^{\prime}$, and $\vec{v}_{2}^{\prime} \equiv \overrightarrow{\vec{r}}_{2}^{\prime}$, from Eqs. (24) and (26) we find the following conditions

$$
\begin{aligned}
& \vec{v}_{1}+\vec{v}_{2}=\vec{v}_{1}^{\prime}+\vec{v}_{2}^{\prime}, \\
& \left(\vec{v}_{1}\right)^{2}+\left(\vec{v}_{2}\right)^{2}=\left(\vec{v}_{1}^{\prime}\right)^{2}+\left(\vec{v}_{2}^{\prime}\right)^{2}, \\
& \left(\vec{v}_{1}^{\prime}\right)^{2}=\left(\vec{v}_{2}\right)^{2} .
\end{aligned}
$$

These equations respectively represent the conservation laws for momentum and energy during the collision, and the conservation of energy at the entrance and exit of the particle at the two wormhole's mouths (cf. Ref. [11]).

Equations (27) can in principle be solved either directly in the $\vec{r}_{0}, t_{0}$, and $\bar{t}$ variables, or in terms of the velocity variables (for instance, considering $\vec{v}_{1}^{\prime}$ and $\vec{v}_{2}{ }^{\prime}$ as unknowns and $\vec{v}_{1}$ and $\vec{v}_{2}$ as parameters, the number of equations and unknowns in the problem remaining the same). 
Using velocities as our unknowns and introducing the quantities

$$
\begin{aligned}
& \vec{a} \equiv \vec{v}_{1}-\vec{v}_{2}^{\prime}=\vec{v}_{1}^{\prime}-\vec{v}_{2} \\
& \vec{b} \equiv \vec{v}_{1}^{\prime}+\vec{v}_{2} \\
& \vec{c} \equiv \vec{v}_{1}+\vec{v}_{2}^{\prime}
\end{aligned}
$$

Eqs. (27) can be easily transformed into the equivalent system of conditions

$$
\begin{aligned}
& \vec{a} \cdot \vec{b}=0, \\
& \vec{a} \cdot \vec{c}=0 .
\end{aligned}
$$

For the motion in three spatial dimensions, the most general solution of the conservation laws (27) is thus given by

$$
\begin{aligned}
& \vec{v}_{1}=\frac{1}{2}(\vec{c}+\vec{a}), \\
& \vec{v}_{2}=\frac{1}{2}(\vec{b}-\vec{a}), \\
& \vec{v}_{1}^{\prime}=\frac{1}{2}(\vec{b}+\vec{a}), \\
& \vec{v}_{2}^{\prime}=\frac{1}{2}(\vec{c}-\vec{a}),
\end{aligned}
$$

for any arbitrary $\vec{a}$ which is orthogonal to arbitrary $\vec{b}$ and $\vec{c}$. Then, in principle, in the case of a generic three dimensional motion Eqs. (29) (with $\vec{a}$ given by the first of Eqs. (28)) can be solved, using Eqs. (24), for $\vec{r}_{0}, t_{0}$, and $\bar{t}$ and therefore for the complete trajectories. We shall not do that here, but only consider the simpler case of two dimensional spatial motion.

\subsubsection{Coplanar motion}

The solutions of the conservation Eqs. (27) for the case of two dimensional, coplanar motion of the copies of the particle with respect to the wormhole's mouths can be deduced from the generic three dimensional solutions (30) by restricting to the following ansätze for $\vec{a}, \vec{b}$ and $\vec{c}$

$$
\text { i) } \vec{a}=\overrightarrow{0} ; \text { or ii) } \vec{c}=\epsilon \vec{b}
$$

where $\epsilon$ is an arbitrary constant.

In particular, the ansatz i) of Eq. (31) corresponds to the case of

\section{a) 'Velocity exchange' rule:}

$$
\begin{aligned}
& \vec{v}_{1}^{\prime}=\vec{v}_{2}, \\
& \vec{v}_{2}^{\prime}=\vec{v}_{1},
\end{aligned}
$$

while the ansatz ii) of Eq. (31) (for $\epsilon \neq-1$ ) corresponds to the case of 


\section{b) 'Mirror exchange of velocities' rule:}

$$
\begin{aligned}
& \left(v_{1}^{\prime}\right)_{x}=\left(v_{2}\right)_{x} \\
& \left(v_{2}^{\prime}\right)_{x}=\left(v_{1}\right)_{x} \\
& \left(v_{1}^{\prime}\right)_{y}=-\left(v_{2}\right)_{y} \\
& \left(v_{2}^{\prime}\right)_{y}=-\left(v_{1}\right)_{y}
\end{aligned}
$$

(expressed in component notation, where we have chosen the $x$-axis to lie along the direction of $\vec{v}_{1}+\vec{v}_{2}=\vec{v}_{1}^{\prime}+\vec{v}_{2}^{\prime}$ ).

For the particular value $\epsilon=-1$, the ansatz ii) of Eq. (31) no longer corresponds to the 'topology' of the 'mirror exchange solutions,' but to the case of

\section{c) 'Collinear velocities' rule: []}

$$
\begin{aligned}
& \vec{v}_{1}=-\vec{v}_{2} \\
& \vec{v}_{1}^{\prime}=-\vec{v}_{2}^{\prime} \\
& \left|\vec{v}_{1}\right|=\left|\vec{v}_{2}\right|=\left|\vec{v}_{1}^{\prime}\right|=\left|\vec{v}_{2}^{\prime}\right| .
\end{aligned}
$$

These solutions are 'degenerate' in the sense that the velocities $\vec{v}_{1}$ and $\vec{v}_{2}$ must be along the direction identified by $\vec{r}_{i}$ and $\vec{r}_{A}$ (i.e. the velocity of the first copy of the particle must be initially pointing towards the wormhole mouth A), and similarly the velocities $\vec{v}_{1}^{\prime}$ and $\vec{v}_{2}^{\prime}$ must be along the direction identified by $\vec{r}_{f}$ and $\vec{r}_{B}$ (i.e. the velocity of the second copy of the particle after the collision must be outwards pointing from the wormhole mouth B). 9 It is also easy to show that the velocity rules (34), combined with the condition that the total duration of the motion (as seen from an observer external and at rest with respect to the wormhole) is fixed and equal to $t_{f}-t_{i}+\tau$, limit the possible choices of the boundary data $\vec{r}_{i}, \vec{r}_{f}, t_{i}, t_{f}$. In particular, only five of such data (e.g., $\vec{r}_{i}, t_{i}$ and $\vec{r}_{f}$ ) can be arbitrarily chosen, while the sixth (e.g. $\left.t_{f}\right)$ will be constrained.

Finally, there is also the 'trivial' solution in which the velocities of the copies of the particle do not change before and after the collision. This actually corresponds to the no-collision case which we already considered in Section 3.1.

\subsubsection{Stationary points for the coplanar motion}

Let us consider the three nontrivial solutions of the conservation Eqs. (27).

As they stand, solutions (32)-(34) are still implicit equations for the original variables $\vec{r}_{0}, t_{0}$ and $\bar{t}$, which are the final object of our analysis of the stationary point

\footnotetext{
${ }^{d}$ This case apparently was not considered in Ref. [11].

'The solution $\vec{c}=-\vec{b}, \vec{a}=\overrightarrow{0}$ is a 'doubly degenerate' case, as it corresponds to one dimensional spatial motion of the two copies of the particle along the line connecting the wormhole's mouths.
} 
of the action (4). The algebra leading to the 'solutions' for the velocity rules $a-c$ in terms of the collision coordinates and $\bar{t}$ is not particularly interesting and we leave the details for the interested reader in the Appendix A.2.

The main result of such an analysis is that, for each of the cases $a-c$, there exists a unique solution for which the action (26) is stationary and minimized with respect to the parameters $\vec{r}_{0}, t_{0}$, and $\bar{t}$ (the stationary values of $t_{0}, \bar{t}$, and $\vec{r}_{0}$ in each of the three cases are respectively given by Eqs. (40)-(42) for case $a$, Eqs. (50), (51), and (53) for case $b$, and finally Eqs. (60)-(61) for case $c$ ). In other words, if we fix the initial and final conditions (2) and the boundary conditions (3), the variational problem for the action (ष), subject to the condition of self-collision for the particle, admits only the set of globally self-consistent classical trajectories given by Eqs. (43)-(44), (54), (62), each of which is subject, respectively, to the constraints (48), (52) and (57), (63) and (65).

We have thus proved that, for the model of a particle which is constrained to traverse a wormhole time-machine geometry once and to self-interact via the 'hardsphere' potential (11), the whole set of classical trajectories which are globally selfconsistent can be directly and simply recovered by imposing the principle of minimal action.

\section{Discussion}

If the laws of physics actually permit the existence of traversible wormholes, then in principle it would be possible to convert the wormhole into a time machine and therefore have CTCs looping through it.

The analysis of the present paper is purely classical. We have considered the simple model of a nonrelativistic particle which is constrained to have fixed initial and final positions, to loop through the wormhole once and to interact with itself by means of a 'hard-sphere', elastic potential $V$. We used the action principle to derive the classical trajectories, and we found that the only possible solutions which minimize the action are those which are globally self-consistent. In the case of coplanar motion with respect to the wormhole's mouths, the possible, globally self-consistent trajectories in which the particle's copies collide are of three types, depending on the three possible ways in which momentum is exchanged (i.e., 'velocity', 'mirror' or 'collinear' exchange) at the collision event. In this way we have shown that the 'Principle of self-consistency' can be actually encoded in a natural way into the 'Principle of minimal action'.

In a previous series of papers [11, 12], the analogous model for the motion of a nonrelativistic 'billiard ball'-like particle moving in the spacetime containing a wormhole time machine has been considered in the context of a Cauchy initial value problem (for a general discussion also including the case of a scalar field, see Ref. [9]). In the case of elastic, no-frictional self-interaction between the two copies of the particle, it has been shown [11] that generic classes of initial data have multiple, and even infinite 
numbers of globally self-consistent solutions to the equations of motion (trajectories where the particle is initially at rest far from the wormhole have, in fact, multiplicity one), with no evidence for non self-consistent trajectories. The extension to the case of an inelastic self-collision of 'billiard ball'-like particles was made in Ref. [12].

Our globally self-consistent solutions for the case of collision with 'velocity exchange' and 'mirror exchange of velocities' are in fact consistent with the solutions found in Ref. [11]. A more detailed analysis of the multiplicity of solutions and the connection between the Cauchy and the boundary value problems, together with a consideration of the back-reaction effects of the particle motion on the wormhole geometry, and of more complicated self-interaction potentials for the particle itself, will be made in a future publication.

In this paper we have not addressed the analysis of the conditions under which a wormhole can be created and maintained in a Lorentzian spacetime (see also Ref. [18]). It is known that static wormholes require a matter which violates the averaged weak energy condition (AWEC) $[3,5]$ (and if spacetime has to maintain a well defined spinorial structure on it, then the creation of wormholes should also occur in pairs [19). Classically, this condition might be a problem, since ordinary classical energy densities are positive."

On the other hand, it is not clear whether quantum effects could anyway preserve the AWEC for generic cases (a well known counterexample is the Casimir effect). Whether vacuum polarization effects in the quantum theory can actually destabilize a wormhole (due to the infinite back-reaction caused by a diverging renormalized stress-energy tensor) is still a challenging issue (for two approaches with opposite conclusions see, e.g., Refs. [21, 22]).

The results of this paper motivate us to formulate the conjecture that the 'Principle of self-consistency' is a consequence of the 'Principle of minimal action' in the general case for all physical phenomena, and not only for the simple mechanical problem considered here.

\section{Acknowledgements}

This work is supported in part by Danish Natural Science Research Council through grant N9401635 and in part by Danmarks Grundforskningsfond through its support for the establishment of the Theoretical Astrophysics Center. A.C.'s research is supported by an EEC fellowship in the 'Human Capital and Mobility' program, under contract No. ERBCHBICT930313.

\footnotetext{
${ }^{f}$ A new interesting set of dynamical wormhole solutions has been recently discovered 20 which satisfy the weak and dominant energy conditions, although it is not clear that these wormholes can be traversed.
} 


\section{A Appendix}

\section{A.1 The 'hard-sphere' potential}

In this appendix we prove some interesting properties for the 'hard-sphere' potential of Eq. (11).

\section{- Energy conservation}

For the potential (11) the second of conservation Eqs. (9) is apparently ill defined at the collision event. What, however, this equation clearly says is that, in the region $r<r_{s}$, the motion is not classically allowed, as the kinetic energy for $\vec{r}$ would be infinitely large and negative. Assuming that the classical motion proceeds until $r=r_{s}$ (i.e., there is the collision at time $t_{0}$ ), we can now integrate both sides of the second of Eqs. (8) around $r_{s}$, obtaining

$$
\begin{aligned}
\frac{I}{m} & \doteq \int_{r_{s}+\epsilon_{1}}^{r_{s}} d \vec{r} \cdot \ddot{\vec{r}}+\int_{r_{s}}^{r_{s}+\epsilon_{2}} d \vec{r} \cdot \ddot{\vec{r}} \\
& =\int_{t_{0}-\delta t_{1}}^{t_{0}} d t \frac{d}{d t}(\dot{\vec{r}})^{2}+\int_{t_{0}}^{t_{0}+\delta t_{2}} d t \frac{d}{d t}(\dot{\vec{r}})^{2}=\left[\dot{\vec{r}}\left(t_{0}+\delta t_{2}\right)\right]^{2}-\left[\dot{\vec{r}}\left(t_{0}-\delta t_{1}\right)\right]^{2}
\end{aligned}
$$

(with $\epsilon_{1}, \epsilon_{2}, \delta t_{1}, \delta t_{2}>0$ ) when acting on the left hand side, while

$$
I=2 V_{1} \int_{r_{s}+\epsilon_{1}}^{r_{s}+\epsilon_{2}} d \vec{r} \cdot\left[\frac{\vec{r}}{r} \delta\left(r-r_{s}\right)\right]=2 V_{1} \int_{r_{s}+\epsilon_{1}}^{r_{s}+\epsilon_{2}} d r \delta\left(r-r_{s}\right)=0
$$

when acting on the right hand side. From Eqs. (35) and (36) we conclude that the kinetic energy $\dot{\vec{r}}^{2}$ is conserved before and after the collision, as expected.

- Zero contribution to classical action

Let us consider the contribution of the potential (11) to the action (4) evaluated along the classical trajectories (15) or (24).

In the no-collision case, denoting with $t_{m}$ and $r_{m}$ the time and position of minimum distance between the two copies of the particle, we have, for the potential (11)

$$
\begin{aligned}
\left.\int_{\bar{t}}^{\bar{t}+\tau} d t V(r)\right|_{c l} & =V_{1}\left[\int_{\bar{t}}^{t_{m}} d t \theta\left(r_{s}-r\right)+\int_{t_{m}}^{\bar{t}+\tau} d t \theta\left(r_{s}-r\right)\right] \\
& =V_{1}\left[\int_{r(\bar{t})}^{r_{m}} \frac{d r}{\dot{r}} \theta\left(r_{s}-r\right)+\int_{r_{m}}^{r(\bar{t}+\tau)} \frac{d r}{\dot{r}} \theta\left(r_{s}-r\right)\right]=0
\end{aligned}
$$

since obviously, in the no-collision case, $r_{m}, r(\bar{t})$ and $r(\bar{t}+\tau)$ are always greater than $r_{s}$.

Similarly, in the collision case, in the limit $r_{s} \rightarrow 0$, the motion is such that (except at the point of collision) the angular part $(\dot{\theta})$ of the velocity $\dot{\vec{r}}$ is essentially zero, and

${ }^{g}$ These results are valid for the generic case of motion in three spatial dimensions. 
using this result together with the conservation equation for $\dot{\vec{r}}^{2}$ gives again

$$
\begin{aligned}
\left.\int_{\bar{t}}^{\bar{t}+\tau} d t V(r)\right|_{c l} & =V_{1}\left[\int_{\bar{t}}^{t_{0}} d t \theta\left(r_{s}-r\right)+\int_{t_{0}}^{\bar{t}+\tau} d t \theta\left(r_{s}-r\right)\right] \\
& \simeq \frac{V_{1}}{\dot{r}}\left[\int_{r(\bar{t})}^{r_{s}} d r \theta\left(r_{s}-r\right)+\int_{r_{s}}^{r(\bar{t}+\tau)} d r \theta\left(r_{s}-r\right)\right]=0
\end{aligned}
$$

In conclusion, the contribution of the 'hard-sphere' potential $V$ to the classical action can always be neglected both in the collision and no-collision cases.

\section{A.2 Trajectories for the coplanar motion}

- 'Velocity exchange'

For the case of 'velocity exchange' between the two copies of the particle at the collision event, the solutions (32) can be rewritten, using Eqs. (24), in terms of the variables $\vec{r}_{0}, t_{0}$ and $\bar{t}$ as

$$
\begin{aligned}
& 0=\frac{\left(\vec{r}_{0}-\vec{r}_{i}\right)}{\left(t_{0}-t_{i}\right)}+\frac{\left(\vec{r}_{0}-\vec{r}_{f}\right)}{\left(t_{f}-t_{0}\right)} \\
& 0=\frac{\left(\vec{r}_{0}-\vec{r}_{A}\right)}{\left(t_{0}-\bar{t}\right)}+\frac{\left(\vec{r}_{0}-\vec{r}_{B}\right)}{\left(\bar{t}+\tau-t_{0}\right)}
\end{aligned}
$$

We can solve this system by first writing, from the first of Eqs. (39), $\vec{r}_{0}$ as a function of $t_{0}$ and then inserting this result into the second of Eqs. (39). We thus obtain a set of two equations (one for each component of the vectors $\vec{r}_{\alpha}$, with $\alpha=0, i, f, A, B$ ) which can be solved in terms of $\bar{t}$ and $t_{0}$, giving

$$
t_{0}=\frac{\left\{\left[\left(\vec{r}_{B}-\vec{r}_{A}\right) \wedge\left(t_{f} \vec{r}_{i}-t_{i} \vec{r}_{f}\right)\right]_{3}+\left[\vec{r}_{A} \wedge \vec{r}_{B}\right]_{3}\left(t_{f}-t_{i}\right)\right\}}{\left[\left(\vec{r}_{f}-\vec{r}_{i}\right) \wedge\left(\vec{r}_{B}-\vec{r}_{A}\right)\right]_{3}}
$$

and

$$
\begin{aligned}
\bar{t} & =\left\{\left[\left(\vec{r}_{B}-\vec{r}_{A}\right) \wedge\left(t_{f} \vec{r}_{i}-t_{i} \vec{r}_{f}\right)\right]_{3}+\left[\vec{r}_{A} \wedge \vec{r}_{B}\right]_{3}\left(t_{f}-t_{i}\right)\right. \\
& \left.\left.+\left[\vec{r}_{i} \wedge \vec{r}_{f}+\left(\vec{r}_{f}-\vec{r}_{i}\right) \wedge \vec{r}_{A}\right)\right]_{3} \tau\right\} \cdot\left\{\left[\left(\vec{r}_{f}-\vec{r}_{i}\right) \wedge\left(\vec{r}_{B}-\vec{r}_{A}\right)\right]_{3}\right\}^{-1}
\end{aligned}
$$

where we have 'artificially' defined vectors in three-dimensional space (i.e., we define $\left.\vec{r}_{\alpha} \doteq\left(x_{\alpha}, y_{\alpha}, 0\right)\right)$, and the notation $]_{3}$ means the third component of the vector.

Substituting these results into Eq. (39) then also explicitly gives the spatial coordinates of the collision event, i.e.

$$
\vec{r}_{0}=\frac{\left\{\left(\vec{r}_{f}-\vec{r}_{i}\right)\left[\vec{r}_{A} \wedge \vec{r}_{B}\right]_{3}+\vec{r}_{i}\left[\vec{r}_{f} \wedge\left(\vec{r}_{B}-\vec{r}_{A}\right)\right]_{3}-\vec{r}_{f}\left[\vec{r}_{i} \wedge\left(\vec{r}_{B}-\vec{r}_{A}\right)\right]_{3}\right\}}{\left[\left(\vec{r}_{f}-\vec{r}_{i}\right) \wedge\left(\vec{r}_{B}-\vec{r}_{A}\right)\right]_{3}}
$$

Finally, we can insert the formulas (40)-(42) into Eqs. (24) to find out the classical trajectories as

$$
\vec{r}_{1}(t)=\vec{r}_{2}^{\prime}(t)=\frac{\left[\vec{r}_{i}\left(t_{f}-t\right)+\vec{r}_{f}\left(t-t_{i}\right)\right]}{\left(t_{f}-t_{i}\right)}
$$


and

$$
\begin{aligned}
\vec{r}_{1}^{\prime}(t) & =\vec{r}_{2}(t)=\vec{r}_{A}+\left\{\left(\vec{r}_{A}-\vec{r}_{B}\right)\left[\left(\vec{r}_{B}-\vec{r}_{A}\right) \wedge\left(t_{f} \vec{r}_{i}-t_{i} \vec{r}_{f}\right)\right]_{3}\right. \\
& +\left[\vec{r}_{A} \wedge \vec{r}_{B}\right]_{3}\left(t_{f}-t_{i}\right)+\left[\vec{r}_{i} \wedge \vec{r}_{f}+\left(\vec{r}_{f}-\vec{r}_{i}\right) \wedge \vec{r}_{A}\right]_{3} \tau \\
& \left.+\left[\left(\vec{r}_{B}-\vec{r}_{A}\right) \wedge\left(\vec{r}_{f}-\vec{r}_{i}\right)\right]_{3} t\right\} \cdot\left\{\tau\left[\left(\vec{r}_{f}-\vec{r}_{i}\right) \wedge\left(\vec{r}_{B}-\vec{r}_{A}\right)\right]_{3}\right\}^{-1}
\end{aligned}
$$

Similarly as done for the case of no-collision, we also have to check that the solutions for $t_{0}$ and $\bar{t}$ given by Eqs. (40)-(41) satisfy the conditions

$$
\begin{aligned}
t_{i} & <\bar{t}<t_{f}-\tau \\
\bar{t} & <t_{0}<\bar{t}+\tau
\end{aligned}
$$

The first of conditions (45) implies

$$
\begin{aligned}
& 0< \frac{\left\{\left[\left(\vec{r}_{B}-\vec{r}_{A}\right) \wedge \vec{r}_{i}+\vec{r}_{A} \wedge \vec{r}_{B}\right]_{3}\left(t_{f}-t_{i}\right)+\left[\vec{r}_{i} \wedge \vec{r}_{f}+\left(\vec{r}_{f}-\vec{r}_{i}\right) \wedge \vec{r}_{A}\right]_{3} \tau\right\}}{\left[\left(\vec{r}_{f}-\vec{r}_{i}\right) \wedge\left(\vec{r}_{B}-\vec{r}_{A}\right)\right]_{3}} \\
& 0>\frac{\left\{\left[\left(\vec{r}_{B}-\vec{r}_{A}\right) \wedge \vec{r}_{f}+\vec{r}_{A} \wedge \vec{r}_{B}\right]_{3}\left(t_{f}-t_{i}\right)+\left[\vec{r}_{i} \wedge \vec{r}_{f}+\left(\vec{r}_{f}-\vec{r}_{i}\right) \wedge \vec{r}_{B}\right]_{3} \tau\right\}}{\left[\left(\vec{r}_{f}-\vec{r}_{i}\right) \wedge\left(\vec{r}_{B}-\vec{r}_{A}\right)\right]_{3}}
\end{aligned}
$$

while the second of conditions (45) implies

$$
\begin{array}{r}
0>\frac{\left[\vec{r}_{i} \wedge \vec{r}_{f}+\left(\vec{r}_{f}-\vec{r}_{i}\right) \wedge \vec{r}_{A}\right]_{3}}{\left[\left(\vec{r}_{f}-\vec{r}_{i}\right) \wedge\left(\vec{r}_{B}-\vec{r}_{A}\right)\right]_{3}} \\
0<\frac{\left[\vec{r}_{i} \wedge \vec{r}_{f}+\left(\vec{r}_{f}-\vec{r}_{i}\right) \wedge \vec{r}_{B}\right]_{3}}{\left[\left(\vec{r}_{f}-\vec{r}_{i}\right) \wedge\left(\vec{r}_{B}-\vec{r}_{A}\right)\right]_{3}}
\end{array}
$$

Equations (46) and (47) can be simultaneously satisfied for

$$
\begin{aligned}
& 0<\left[\vec{r}_{i} \wedge \vec{r}_{f}+\left(\vec{r}_{f}-\vec{r}_{i}\right) \wedge \vec{r}_{A}\right]_{3} \\
& 0>\left[\vec{r}_{i} \wedge \vec{r}_{f}+\left(\vec{r}_{f}-\vec{r}_{i}\right) \wedge \vec{r}_{B}\right]_{3} \\
& 0>\left[\left(\vec{r}_{B}-\vec{r}_{A}\right) \wedge \vec{r}_{i}+\vec{r}_{A} \wedge \vec{r}_{B}\right]_{3}\left(t_{f}-t_{i}\right)+\left[\vec{r}_{i} \wedge \vec{r}_{f}+\left(\vec{r}_{f}-\vec{r}_{i}\right) \wedge \vec{r}_{A}\right]_{3} \tau \\
& 0<\left[\left(\vec{r}_{B}-\vec{r}_{A}\right) \wedge \vec{r}_{f}+\vec{r}_{A} \wedge \vec{r}_{B}\right]_{3}\left(t_{f}-t_{i}\right)+\left[\vec{r}_{i} \wedge \vec{r}_{f}+\left(\vec{r}_{f}-\vec{r}_{i}\right) \wedge \vec{r}_{B}\right]_{3} \tau
\end{aligned}
$$

or, equivalently, if all the inequality signs in (48) are reversed. Conditions (48) are thus the constraints on the classical trajectory for the 'velocity exchange' rule.

- 'Mirror exchange of velocities'

The main lines of the analysis for the 'mirror exchange of velocities' case are essentially the same as for the 'velocity exchange' case. Here, however, due to the rules (33), it is more convenient to use a somewhat more involved component notation, where all vector quantities are expressed as $\vec{r}_{\alpha} \doteq\left(x_{\alpha}, y_{\alpha}\right)$.

Using this notation and Eqs. (24), the rules (33) in terms of $x_{0}, y_{0}, t_{0}$ and $\bar{t}$ explicitly become

$$
0=\frac{\left(x_{0}-x_{B}\right)}{\left(\bar{t}+\tau-t_{0}\right)}+\frac{\left(x_{0}-x_{A}\right)}{\left(t_{0}-\bar{t}\right)}
$$




$$
\begin{aligned}
0 & =\frac{\left(x_{0}-x_{f}\right)}{\left(t_{f}-t_{0}\right)}+\frac{\left(x_{0}-x_{i}\right)}{\left(t_{0}-t_{i}\right)} \\
0 & =\frac{\left(y_{0}-y_{B}\right)}{\left(\bar{t}+\tau-t_{0}\right)}-\frac{\left(y_{0}-y_{A}\right)}{\left(t_{0}-\bar{t}\right)} \\
0 & =\frac{\left(y_{0}-y_{f}\right)}{\left(t_{f}-t_{0}\right)}-\frac{\left(y_{0}-y_{i}\right)}{\left(t_{0}-t_{i}\right)} \\
0 & =\frac{\left(y_{0}-y_{i}\right)}{\left(t_{0}-t_{i}\right)}+\frac{\left(y_{0}-y_{A}\right)}{\left(t_{0}-\bar{t}\right)}
\end{aligned}
$$

The last of Eqs. (49) is an extra constraint which must be imposed in the particular coordinate frame that we are using, for which we have that $\left(v_{1}\right)_{y}+\left(v_{2}\right)_{y}=0$.

From the second and fourth of conditions (49), we can solve for $x_{0}$ and $y_{0}$ as functions of $t_{0}$, and inserting these results into the remaining equations of the system (49) we get three equations for the two 'unknowns' $t_{0}$ and $\bar{t}$. This means that the trajectory will be constrained as in the 'velocity exchange' case.

The explicit stationary solutions for $t_{0}$ and $\bar{t}$ are

$$
\begin{aligned}
t_{0}= & t_{i}+\frac{\left[x_{A}\left(y_{i}-y_{B}\right)+x_{B}\left(y_{i}-y_{A}\right)+x_{i}\left(y_{A}+y_{B}-2 y_{i}\right)\right]\left(t_{f}-t_{i}\right)}{\left[\left(x_{B}-x_{A}\right)\left(y_{A}-y_{B}\right)\left(t_{f}-t_{i}\right)+\left(x_{f}-x_{i}\right)\left(2 y_{i}-y_{A}-y_{B}\right) \tau\right]} \tau \\
\bar{t} & =t_{i}+\tau\left\{\left[x_{A}\left(y_{A}+y_{i}-2 y_{B}\right)+x_{B}\left(y_{i}-y_{A}\right)+2 x_{i}\left(y_{B}-y_{i}\right)\right]\left(t_{f}-t_{i}\right)\right. \\
& \left.+\left(x_{f}-x_{i}\right)\left(y_{A}-y_{i}\right) \tau\right\}\left\{\left(x_{A}-x_{B}\right)\left(y_{B}-y_{A}\right)\left(t_{f}-t_{i}\right)\right. \\
& \left.+\left(x_{f}-x_{i}\right)\left(2 y_{i}-y_{A}-y_{B}\right) \tau\right\}^{-1}
\end{aligned}
$$

while the constraint is

$$
\begin{aligned}
0 & =\tau^{2}\left(x_{f}-x_{i}\right)\left(y_{f}-y_{i}\right)+\tau\left(t_{f}-t_{i}\right)\left[\left(x_{i}+x_{f}\right)\left(y_{A}+y_{B}\right)-2\left(x_{i} y_{f}+x_{f} y_{i}\right)\right. \\
& \left.+\left(x_{A}+x_{B}\right)\left(y_{i}+y_{f}\right)-2\left(x_{A} y_{B}+x_{B} y_{A}\right)\right]+\left(t_{f}-t_{i}\right)^{2}\left(x_{A}-x_{B}\right)\left(y_{A}-y_{B}\right)(52
\end{aligned}
$$

Using Eqs. (50)-(51) back into the system (49), we finally find the spatial coordinates of the collision event as

$$
\begin{aligned}
x_{0} & =x_{i}+\frac{\left[x_{A}\left(y_{i}-y_{B}\right)+x_{B}\left(y_{i}-y_{A}\right)+x_{i}\left(y_{A}+y_{B}-2 y_{i}\right)\right]\left(x_{f}-x_{i}\right)}{\left[\left(x_{B}-x_{A}\right)\left(y_{A}-y_{B}\right)\left(t_{f}-t_{i}\right)+\left(x_{f}-x_{i}\right)\left(2 y_{i}-y_{A}-y_{B}\right) \tau\right]} \tau \\
y_{0} & =y_{i}+\left\{\left[x_{A}\left(y_{B}-y_{i}\right)+x_{B}\left(y_{A}-y_{i}\right)+x_{i}\left(2 y_{i}-y_{A}-y_{B}\right)\right]\left(y_{f}-y_{i}\right) \tau\right\} \\
& \times\left\{\left(x_{B}-x_{A}\right)\left(y_{A}-y_{B}\right)\left(t_{f}-t_{i}\right)+\left[2 x_{A}\left(y_{B}-y_{i}\right)\right.\right. \\
& \left.\left.+2 x_{B}\left(y_{A}-y_{i}\right)+\left(x_{f}+x_{i}\right)\left(2 y_{i}-y_{A}-y_{B}\right)\right] \tau\right\}^{-1}
\end{aligned}
$$

and, using Eqs. (50)-(51) and (53), the trajectories (24) explicitly become

$$
x_{1}=x_{i}+\frac{\left(x_{f}-x_{i}\right)}{\left(t_{f}-t_{i}\right)}\left(t-t_{i}\right)
$$




$$
\begin{aligned}
& y_{1}=y_{i}+\left\{\left[\left(x_{A}-x_{B}\right)\left(y_{A}-y_{B}\right)\left(t_{f}-t_{i}\right)+\left(x_{f}-x_{i}\right)\left(y_{A}+y_{B}-2 y_{i}\right) \tau\right]\right. \\
& \left.\times\left(y_{f}-y_{i}\right)\left(t-t_{i}\right)\right\}\left\{\left(x_{B}-x_{A}\right)\left(y_{A}-y_{B}\right)\left(t_{f}-t_{i}\right)+\left[2 x_{A}\left(y_{B}-y_{i}\right)\right.\right. \\
& \left.\left.+2 x_{B}\left(y_{A}-y_{i}\right)+\left(x_{f}+x_{i}\right)\left(2 y_{i}-y_{A}-y_{B}\right) \tau\right]\right\}^{-1} \\
& x_{2}=x_{A}+\left(x_{B}-x_{A}\right)\left\{\frac{\left(t-t_{i}\right)}{\tau}+\left\{\left(x_{f}-x_{i}\right)\left(y_{i}-y_{A}\right) \tau\right.\right. \\
& \left.+\left[x_{A}\left(2 y_{B}-y_{A}-y_{i}\right)+x_{B}\left(y_{A}-y_{i}\right)+2 x_{i}\left(y_{i}-y_{B}\right)\right]\left(t_{f}-t_{i}\right)\right\} \\
& \left.\times\left[\left(x_{B}-x_{A}\right)\left(y_{A}-y_{B}\right)\left(t_{f}-t_{i}\right)+\left(x_{f}-x_{i}\right)\left(2 y_{i}-y_{A}-y_{B}\right) \tau\right]^{-1}\right\} \\
& y_{2}=y_{A}+\left\{\left(x_{B}-x_{A}\right)\left(y_{A}-y_{B}\right)\left(y_{i}-y_{A}\right)\left(t_{f}-t_{i}\right)+\left[\left[x_{A}\left(y_{B}-y_{i}\right)\right.\right.\right. \\
& \left.+x_{B}\left(y_{A}-y_{i}\right)\right]\left(y_{i}+y_{f}-2 y_{A}\right)+\left[x_{f}\left(y_{i}-y_{A}\right)+x_{i}\left(y_{f}-y_{A}\right)\right] \\
& \left.\left.\times\left(2 y_{i}-y_{A}-y_{B}\right)\right] \tau\right\}\left\{\left[x_{A}\left(2 y_{B}-y_{A}-y_{i}\right)+x_{B}\left(y_{A}-y_{i}\right)\right.\right. \\
& \left.+2 x_{i}\left(y_{i}-y_{B}\right)\right]\left(t_{f}-t_{i}\right)+\left(x_{f}-x_{i}\right)\left(y_{i}-y_{A}\right) \tau \\
& \left.+\left[\left(x_{B}-x_{A}\right)\left(y_{A}-y_{B}\right)\left(t_{f}-t_{i}\right)+\left(x_{f}-x_{i}\right)\left(2 y_{i}-y_{A}-y_{B}\right) \tau\right] \frac{\left(t-t_{i}\right)}{\tau}\right\} \\
& \times\left\{\left(x_{A}-x_{i}\right)\left(y_{B}-y_{A}\right)\left(t_{f}-t_{i}\right)+\left(x_{f}-x_{i}\right)\left(y_{i}-y_{A}\right) \tau\right\}^{-1} \\
& \times\left\{\left(x_{B}-x_{A}\right)\left(y_{A}-y_{B}\right)\left(t_{f}-t_{i}\right)+\left[2 x_{A}\left(y_{B}-y_{i}\right)\right.\right. \\
& \left.\left.+2 x_{B}\left(y_{A}-y_{i}\right)+\left(x_{f}+x_{i}\right)\left(2 y_{i}-y_{A}-y_{B}\right) \tau\right]\right\}^{-1} \\
& x_{1}^{\prime}=x_{B}+\left(x_{B}-x_{A}\right)\left\{\frac{\left(t-t_{i}\right)}{\tau}\right. \\
& \left.+\frac{\left[\left(x_{A}+x_{B}-2 x_{i}\right)\left(t_{f}-t_{i}\right)+\left(x_{f}-x_{i}\right) \tau\right]\left(y_{B}-y_{i}\right)}{\left[\left(x_{B}-x_{A}\right)\left(y_{A}-y_{B}\right)\left(t_{f}-t_{i}\right)+\left(x_{f}-x_{i}\right)\left(2 y_{i}-y_{A}-y_{B}\right) \tau\right]}\right\} \\
& y_{1}^{\prime}=y_{B}+\left\{\left(x_{B}-x_{A}\right)\left(y_{A}-y_{B}\right)\left(y_{i}-y_{B}\right)\left(t_{f}-t_{i}\right)+\left[\left[x_{A}\left(y_{B}-y_{i}\right)\right.\right.\right. \\
& \left.+x_{B}\left(y_{A}-y_{i}\right)\right]\left(y_{i}+y_{f}-2 y_{B}\right)+\left[x_{f}\left(y_{i}-y_{B}\right)+x_{i}\left(y_{f}-y_{B}\right)\right] \\
& \left.\left.\times\left(2 y_{i}-y_{A}-y_{B}\right)\right] \tau\right\}\left\{\left[\left(x_{A}+x_{B}-2 x_{i}\right)\left(t_{f}-t_{i}\right)+\left(x_{f}-x_{i}\right) \tau\right]\left(y_{i}-y_{B}\right)\right. \\
& \left.+\left[\left(x_{B}-x_{A}\right)\left(y_{B}-y_{A}\right)\left(t_{f}-t_{i}\right)+\left(x_{f}-x_{i}\right)\left(y_{A}+y_{B}-2 y_{i}\right) \tau\right] \frac{\left(t-t_{i}\right)}{\tau}\right\} \\
& \times\left\{\left(x_{i}-x_{B}\right)\left(y_{B}-y_{A}\right)\left(t_{f}-t_{i}\right)+\left(x_{f}-x_{i}\right)\left(y_{i}-y_{B}\right) \tau\right\}^{-1} \\
& \times\left\{\left(x_{B}-x_{A}\right)\left(y_{A}-y_{B}\right)\left(t_{f}-t_{i}\right)+\left[2 x_{A}\left(y_{B}-y_{i}\right)\right.\right. \\
& \left.\left.+2 x_{B}\left(y_{A}-y_{i}\right)+\left(x_{f}+x_{i}\right)\left(2 y_{i}-y_{A}-y_{B}\right) \tau\right]\right\}^{-1} \\
& x_{2}^{\prime}=x_{f}+\frac{\left(x_{f}-x_{i}\right)}{\left(t_{f}-t_{i}\right)}\left(t-t_{f}\right) \\
& y_{2}^{\prime}=y_{f}+\left\{\left[\left(x_{B}-x_{A}\right)\left(y_{A}-y_{B}\right)\left(t_{f}-t_{i}\right)+\left(x_{f}-x_{i}\right)\left(2 y_{i}-y_{A}-y_{B}\right) \tau\right]\right. \\
& \left.\times\left(y_{f}-y_{i}\right)\left(t-t_{f}\right)\right\}\left\{\left(x_{B}-x_{A}\right)\left(y_{A}-y_{B}\right)\left(t_{f}-t_{i}\right)+\left[2 x_{A}\left(y_{B}-y_{i}\right)\right.\right. \\
& \left.\left.+2 x_{B}\left(y_{A}-y_{i}\right)+\left(x_{f}+x_{i}\right)\left(2 y_{i}-y_{A}-y_{B}\right) \tau\right]\left(t_{f}-t_{i}\right)\right\}^{-1}
\end{aligned}
$$


Imposing the conditions (45) for $t_{0}$ and $\bar{t}$ given by Eqs. (50)-(51), finally gives the following further constraints on the trajectories

$$
\begin{aligned}
0 & >\left\{\tau\left(x_{f}-x_{i}\right)\left(2 y_{i}-y_{B}-y_{f}\right)+\left(t_{f}-t_{i}\right)\left[x_{A}\left(y_{B}-y_{f}\right)\right.\right. \\
& \left.\left.+x_{B}\left(2 y_{A}-y_{B}-y_{f}\right)+2 x_{i}\left(y_{f}-y_{A}\right)\right]\right\} \\
& \times\left[\left(x_{B}-x_{A}\right)\left(y_{A}-y_{B}\right)\left(t_{f}-t_{i}\right)+\left(x_{f}-x_{i}\right)\left(2 y_{i}-y_{A}-y_{B}\right) \tau\right]^{-1} \\
0 & <\left\{\tau\left(x_{f}-x_{i}\right)\left(y_{A}-y_{i}\right)+\left(t_{f}-t_{i}\right)\left[x_{A}\left(y_{A}+y_{i}-2 y_{B}\right)\right.\right. \\
& \left.\left.+x_{B}\left(y_{i}-y_{A}\right)+2 x_{i}\left(y_{B}-y_{i}\right)\right]\right\} \\
& \times\left[\left(x_{B}-x_{A}\right)\left(y_{A}-y_{B}\right)\left(t_{f}-t_{i}\right)+\left(x_{f}-x_{i}\right)\left(2 y_{i}-y_{A}-y_{B}\right) \tau\right]^{-1}
\end{aligned}
$$

and

$$
\begin{aligned}
& 0< \frac{\left[\left(x_{A}-x_{i}\right)\left(y_{B}-y_{A}\right)\left(t_{f}-t_{i}\right)+\left(x_{f}-x_{i}\right)\left(y_{i}-y_{A}\right) \tau\right]}{\left[\left(x_{B}-x_{A}\right)\left(y_{A}-y_{B}\right)\left(t_{f}-t_{i}\right)+\left(x_{f}-x_{i}\right)\left(2 y_{i}-y_{A}-y_{B}\right) \tau\right]} \\
& 0>\frac{\left[\left(x_{B}-x_{i}\right)\left(y_{B}-y_{A}\right)\left(t_{f}-t_{i}\right)+\left(x_{f}-x_{i}\right)\left(y_{B}-y_{i}\right) \tau\right]}{\left[\left(x_{B}-x_{A}\right)\left(y_{A}-y_{B}\right)\left(t_{f}-t_{i}\right)+\left(x_{f}-x_{i}\right)\left(2 y_{i}-y_{A}-y_{B}\right) \tau\right]}
\end{aligned}
$$

which can be satisfied for

$$
\begin{aligned}
0 & <\left(x_{B}-x_{A}\right)\left(y_{A}-y_{B}\right)\left(t_{f}-t_{i}\right)+\left(x_{f}-x_{i}\right)\left(2 y_{i}-y_{A}-y_{B}\right) \tau \\
0 & >\left(t_{f}-t_{i}\right)\left[x_{A}\left(y_{B}-y_{f}\right)+x_{B}\left(2 y_{A}-y_{B}-y_{f}\right)+2 x_{i}\left(y_{f}-y_{A}\right)\right] \\
& +\tau\left(x_{f}-x_{i}\right)\left(2 y_{i}-y_{B}-y_{f}\right) \\
0 & <\left(t_{f}-t_{i}\right)\left[x_{A}\left(y_{A}+y_{i}-2 y_{B}\right)+x_{B}\left(y_{i}-y_{A}\right)+2 x_{i}\left(y_{B}-y_{i}\right)\right] \\
& +\tau\left(x_{f}-x_{i}\right)\left(y_{A}-y_{i}\right) \\
0 & <\left(x_{A}-x_{i}\right)\left(y_{B}-y_{A}\right)\left(t_{f}-t_{i}\right)+\left(x_{f}-x_{i}\right)\left(y_{i}-y_{A}\right) \tau \\
0 & >\left(x_{B}-x_{i}\right)\left(y_{B}-y_{A}\right)\left(t_{f}-t_{i}\right)+\left(x_{f}-x_{i}\right)\left(y_{B}-y_{i}\right) \tau
\end{aligned}
$$

or for the analogous conditions with all the inequality signs reversed.

\section{- 'Collinear velocities'}

For the 'collinear velocities' solution for the conservation Eqs. (27), we can rewrite Eqs. (34), using Eqs. (24), to explicitly show the dependence on $\vec{r}_{0}, t_{0}$ and $\bar{t}$ as

$$
\begin{aligned}
0 & =\frac{\left(\vec{r}_{0}-\vec{r}_{i}\right)}{\left(t_{0}-t_{i}\right)}+\frac{\left(\vec{r}_{0}-\vec{r}_{A}\right)}{\left(t_{0}-\bar{t}\right)} \\
0 & =\frac{\left(\vec{r}_{0}-\vec{r}_{B}\right)}{\left(\bar{t}+\tau-t_{0}\right)}+\frac{\left(\vec{r}_{0}-\vec{r}_{f}\right)}{\left(t_{f}-t_{0}\right)} \\
0 & =\frac{\left(\vec{r}_{0}-\vec{r}_{i}\right)^{2}}{\left(t_{0}-t_{i}\right)^{2}}-\frac{\left(\vec{r}_{0}-\vec{r}_{f}\right)^{2}}{\left(t_{f}-t_{0}\right)^{2}}
\end{aligned}
$$

The algebra involved in the solution of the stationarity problem defined by Eqs. (58) greatly simplifies if we choose to work in the reference frame where the collision event 
is taken as the origin of the spatial coordinates, i.e. $\vec{r}_{0} \doteq(0,0)$, and the $x$-axis is taken along the direction defined by the (collinear) velocities $\vec{v}_{1}$ and $\vec{v}_{2}$ (in other words, along the direction of the vector $\vec{r}_{A}-\vec{r}_{i}$ ). In this frame, the set of conditions (58) is replaced by the much simpler, equivalent system of equations

$$
\begin{aligned}
0 & =y_{A}=y_{i}=x_{0}=y_{0} \\
0 & =\frac{y_{B}}{x_{B}}-\frac{y_{f}}{x_{f}} \\
0 & =\frac{x_{A}}{\left(t_{0}-\bar{t}\right)}+\frac{x_{i}}{\left(t_{0}-t_{i}\right)} \\
0 & =\frac{x_{B}}{\left(\bar{t}+\tau-t_{0}\right)}+\frac{x_{f}}{\left(t_{f}-t_{0}\right)} \\
0 & =\frac{x_{i}^{2}}{\left(t_{0}-t_{i}\right)^{2}}-\frac{\left(x_{f}^{2}+y_{f}^{2}\right)}{\left(t_{f}-t_{0}\right)^{2}}
\end{aligned}
$$

We can easily solve the third and fourth of Eqs. (59) in terms of $t_{0}$ and $\bar{t}$ as

$$
\begin{gathered}
t_{0}=\frac{\left(t_{f} x_{B} x_{i}-t_{i} x_{A} x_{f}+\tau x_{i} x_{f}\right)}{\left(x_{B} x_{i}-x_{A} x_{f}\right)} \\
\bar{t}=\frac{\left[\left(t_{f} x_{B}+\tau x_{f}\right)\left(x_{A}+x_{i}\right)-t_{i} x_{A}\left(x_{f}+x_{B}\right)\right]}{\left(x_{B} x_{i}-x_{A} x_{f}\right)}
\end{gathered}
$$

Moreover, using Eqs. (60)-(61) into Eqs. (24), we can find the classical trajectories as

$$
\begin{aligned}
x_{1}(t) & =-x_{2}(t)=\frac{\left[\left(t_{f}-t\right) x_{B} x_{i}+\left(t-t_{i}\right) x_{A} x_{f}+\tau x_{i} x_{f}\right]}{\left[\left(t_{f}-t_{i}\right) x_{B}+\tau x_{f}\right]} \\
y_{1}(t) & =y_{2}(t)=0 \\
\vec{r}_{1}^{\prime}(t) & =-\frac{\left[\left(t_{f}-t\right) x_{B} x_{i}+\left(t-t_{i}\right) x_{A} x_{f}+\tau x_{i} x_{f}\right]}{\left[\left(t_{f}-t_{i}\right) x_{A}+\tau x_{i}\right] x_{B}} \vec{r}_{B} \\
\vec{r}_{2}^{\prime}(t) & =\frac{\left[\left(t_{f}-t\right) x_{B} x_{i}+\left(t-t_{i}\right) x_{A} x_{f}+\tau x_{i} x_{f}\right]}{\left[\left(t_{f}-t_{i}\right) x_{A}+\tau x_{i}\right] x_{f}} \vec{r}_{f}
\end{aligned}
$$

Besides the 'coordinate-frame' constraints on these trajectories, which are given by the first two of Eqs. (59), and the constraint given by the last of Eqs. (59), which using Eq. (60) becomes

$$
\left|\left[\left(t_{f}-t_{i}\right) x_{A}+\tau x_{i}\right] x_{f}\right|=\sqrt{x_{f}^{2}+y_{f}^{2}}\left|\left(t_{f}-t_{i}\right) x_{B}+\tau x_{f}\right|
$$

we also have the extra constraints which are implied by conditions (45), i.e.

$$
0<\frac{\left(x_{A}+x_{i}\right)\left[\left(t_{f}-t_{i}\right) x_{B}+\tau x_{f}\right]}{\left(x_{B} x_{i}-x_{A} x_{f}\right)}
$$




$$
\begin{aligned}
0 & >\frac{\left(x_{B}+x_{f}\right)\left[\left(t_{f}-t_{i}\right) x_{A}+\tau x_{i}\right]}{\left(x_{B} x_{i}-x_{A} x_{f}\right)} \\
0 & >\frac{x_{A}\left[\left(t_{f}-t_{i}\right) x_{B}+\tau x_{f}\right]}{\left(x_{B} x_{i}-x_{A} x_{f}\right)} \\
0 & <\frac{x_{B}\left[\left(t_{f}-t_{i}\right) x_{A}+\tau x_{i}\right]}{\left(x_{B} x_{i}-x_{A} x_{f}\right)}
\end{aligned}
$$

which can be solved by

$$
\begin{aligned}
& 0<x_{B} x_{i}-x_{A} x_{f} \\
& 0<\left(x_{A}+x_{i}\right)\left[\left(t_{f}-t_{i}\right) x_{B}+\tau x_{f}\right] \\
& 0>\left(x_{B}+x_{f}\right)\left[\left(t_{f}-t_{i}\right) x_{A}+\tau x_{i}\right] \\
& 0>x_{A}\left[\left(t_{f}-t_{i}\right) x_{B}+\tau x_{f}\right] \\
& 0<x_{B}\left[\left(t_{f}-t_{i}\right) x_{A}+\tau x_{i}\right]
\end{aligned}
$$

(or the same formulas with all the inequality signs reversed, as in the previous cases).

\section{A.3 The nature of the stationary points}

In order to study the nature of the stationary points found in the collision cases, we

have to evaluate the eigenvalues of the Hessian $(H)$ of $\hat{S}_{c l, c}$, where $\hat{S}_{c l, c}$ is a function of the variables $\vec{r}_{0}, t_{0}$ and $\bar{t}$.

Taking second order derivatives of the classical action (26) with respect to $x_{0}, y_{0}, t_{0}$ and $\bar{t}$ we find

$$
\begin{aligned}
\left.\frac{\partial^{2} \hat{S}_{c l, c}}{\partial x_{0}^{2}}\right|_{s t a t} & =\left.\frac{\partial^{2} \hat{S}_{c l, c}}{\partial y_{0}^{2}}\right|_{s t a t}=m\left[\frac{1}{\left(t_{0}-t_{i}\right)}+\frac{1}{\left(\bar{t}+\tau-t_{0}\right)}\right. \\
& \left.+\frac{1}{\left(t_{0}-\bar{t}\right)}+\frac{1}{\left(t_{f}-t_{0}\right)}\right]_{s t a t}>0 \\
\left.\frac{\partial^{2} \hat{S}_{c l, c}}{\partial t_{0}^{2}}\right|_{\text {stat }} & =m\left[\frac{\left(\vec{r}_{0}-\vec{r}_{i}\right)^{2}}{\left(t_{0}-t_{i}\right)^{3}}+\frac{\left(\vec{r}_{0}-\vec{r}_{B}\right)^{2}}{\left(\bar{t}+\tau-t_{0}\right)^{3}}+\frac{\left(\vec{r}_{0}-\vec{r}_{A}\right)^{2}}{\left(t_{0}-\bar{t}\right)^{3}}+\frac{\left(\vec{r}_{0}-\vec{r}_{f}\right)^{2}}{\left(t_{f}-t_{0}\right)^{3}}\right]_{s t a t}>0 \\
\left.\frac{\partial^{2} \hat{S}_{c l, c}}{\partial \bar{t}^{2}}\right|_{\text {stat }} & =-\left.\frac{\partial^{2} \hat{S}_{c l, c}}{\partial \bar{t} \partial t_{0}}\right|_{s t a t}=m\left[\frac{\left(\vec{r}_{0}-\vec{r}_{B}\right)^{2}}{\left(\bar{t}+\tau-t_{0}\right)^{3}}+\frac{\left(\vec{r}_{0}-\vec{r}_{A}\right)^{2}}{\left(t_{0}-\bar{t}\right)^{3}}\right]_{s t a t}>0 \\
\left.\frac{\partial^{2} \hat{S}_{c l, c}}{\partial x_{0} \partial t_{0}}\right|_{s t a t} & =m\left[-\frac{\left(x_{0}-x_{i}\right)}{\left(t_{0}-t_{i}\right)^{2}}+\frac{\left(x_{0}-x_{B}\right)}{\left(\bar{t}+\tau-t_{0}\right)^{2}}-\frac{\left(x_{0}-x_{A}\right)}{\left(t_{0}-\bar{t}\right)^{2}}+\frac{\left(x_{0}-x_{f}\right)}{\left(t_{f}-t_{0}\right)^{2}}\right]_{\text {stat }} \\
\left.\frac{\partial^{2} \hat{S}_{c l, c}}{\partial y_{0} \partial t_{0}}\right|_{s t a t} & =m\left[-\frac{\left(y_{0}-y_{i}\right)}{\left(t_{0}-t_{i}\right)^{2}}+\frac{\left(y_{0}-y_{B}\right)}{\left(\bar{t}+\tau-t_{0}\right)^{2}}-\frac{\left(y_{0}-y_{A}\right)}{\left(t_{0}-\bar{t}\right)^{2}}+\frac{\left(y_{0}-y_{f}\right)}{\left(t_{f}-t_{0}\right)^{2}}\right]_{s t a t} \\
\left.\frac{\partial^{2} \hat{S}_{c l, c}}{\partial x_{0} \partial \bar{t}}\right|_{s t a t} & =m\left[-\frac{\left(x_{0}-x_{B}\right)}{\left(\bar{t}+\tau-t_{0}\right)^{2}}+\frac{\left(x_{0}-x_{A}\right)}{\left(t_{0}-\bar{t}\right)^{2}}\right]_{s t a t}
\end{aligned}
$$




$$
\begin{aligned}
\left.\frac{\partial^{2} \hat{S}_{c l, c}}{\partial y_{0} \partial \bar{t}}\right|_{s t a t} & =m\left[-\frac{\left(y_{0}-y_{B}\right)}{\left(\bar{t}+\tau-t_{0}\right)^{2}}+\frac{\left(y_{0}-y_{A}\right)}{\left(t_{0}-\bar{t}\right)^{2}}\right]_{s t a t} \\
\left.\frac{\partial^{2} \hat{S}_{c l, c}}{\partial x_{0} \partial y_{0}}\right|_{s t a t} & =0
\end{aligned}
$$

The Hessian of $\hat{S}_{c l, c}$ is a symmetric $4 \times 4$ matrix with entries given by the expressions (66). The eigenvalues $\lambda$ of $H$ can be found in the standard way by solving for the determinant

$$
\operatorname{Det}\left(\frac{H}{m}-\lambda I\right)=0
$$

which gives the following characteristic equation for $\lambda$

$$
\lambda^{4}+a_{3} \lambda^{3}+a_{2} \lambda^{2}+a_{1} \lambda+a_{0}=0
$$

It can be checked (after a somewhat long and uninteresting algebra which we omit here) that, for all the 'velocity rules' given by Eqs. (32)-(34), the coefficients in Eq. (68) are such that $a_{3}, a_{1}<0$ and $a_{2}, a_{0}>0$, which is enough to show that all eigenvalues $\lambda$ must be positive. Using well known theorems from analysis, we conclude that the stationary point for the action $\hat{S}_{c l, c}$ is a minimum along each of the 4-d hypersurfaces defined by the collision data of cases $a-c$. 


\section{References}

[1] K. Gödel, Rev. Mod. Phys. 21, 447 (1949); C. W. Misner and A. H. Taub, Sov. Phys. JETP 28, 122 (1969).

[2] J. R. Gott III, Phys. Rev. Lett. 66, 1126 (1991).

[3] M. S. Morris and K. S. Thorne, Am. J. Phys. 56, 395 (1988).

[4] M. S. Morris, K. S. Thorne and U. Yurtsever, Phys. Rev. Lett. 61, 1446 (1988).

[5] M. Visser, Phys. Rev. D 39, 3182 (1989); Nucl. Phys. B 328, 203 (1989).

[6] U. Yurtsever, Class. Quantum Grav. 7, L251 (1990); Class. Quantum Grav. 8, 1127 (1991); G. Klinkhammer, Phys. Rev. D 43, 2542 (1991); R. Wald and U. Yurtsever, Phys. Rev. D 44, 403 (1991).

[7] I. D. Novikov, Sov. Phys. JETP 68, 439 (1989).

[8] V. P. Frolov and I. D. Novikov, Phys. Rev. D 42, 1057 (1990).

[9] J. Friedman, M. S. Morris, I. D. Novikov, F. Echeverria, G. Klinkhammer, K. S. Thorne and U. Yurtsever, Phys. Rev. D 42, 1915 (1990).

[10] H. H. Soleng, Class. Quantum Grav. 7, 999 (1990); Gen. Rel. Grav. 24, 111 (1992); Phys. Rev. D 49, 1124 (1994).

[11] F. Echeverria, G. Klinkhammer and K. S. Thorne, Phys. Rev. D 44, 1077 (1991).

[12] E. V. Mikheeva and I. D. Novikov, Phys. Rev. D 47, 1432 (1993).

[13] I. D. Novikov, Evolution of the Universe (Cambridge University Press, Cambridge, 1983); Y. B. Zeldovich and I. D. Novikov, Stroenie i Evolutsia Vselennoi (Nauka, Moscow, 1975).

[14] I. D. Novikov and V. P. Frolov, Physics of Black Holes (Kluwer, New York, 1989).

[15] I. D. Novikov, Phys. Rev. D 45, 1989 (1992).

[16] S. W. Hawking, Nucl. Phys. B 144, 349 (1978).

[17] J. A. Wheeler, Geometrodynamics (Academic Press, New York, 1962).

[18] M. Visser, Phys. Rev. D 41, 1116 (1990).

[19] G. W. Gibbons and S. W. Hawking, Commun. Math. Phys. 148, 345 (1992). 
[20] A. Ori, Phys. Rev. Lett. 71, 2517 (1993); A. Ori and Y. Soen, Phys. Rev. D 49, 3990 (1994); P. S. Letelier and A. Z. Wang, Dynamical wormholes and energy conditions, preprint archive gr/qc-9506004, (1995).

[21] S. W. Kim and K. S. Thorne, Phys. Rev. D 43, 3929 (1991).

[22] S. W. Hawking, Phys. Rev. D 46, 603 (1992); M. Visser, Phys. Lett. B 242, 24 (1990); Phys. Rev. D 43, 402 (1991); Phys. Rev. D 47, 554 (1993); Phys. Rev. D 49, 3963 (1994). 